\title{
GENERATION OF PHASE-CONTROLLED ACCELERATING STRUCTURES IN PLASMA*
}

\author{
$\underline{\text { G. Shvets }}^{\dagger}$, N. J. Fisch, PPPL, Princeton, NJ \\ A. Pukhov, J. Meyer-ter-Vehn, MPQ, Garching, Germany
}

\begin{abstract}
A novel mechanism for generating large $(>1 \mathrm{GeV} / \mathrm{m})$ accelerating wakes in a plasma is considered. We employ two slightly detuned counter-propagating laser beams, an ultra-short timing pulse and a long pump, which exchange photons and deposit the recoil momentum into the plasma electrons. A rapidly rising electron current is thereby generated, inducing an plasma wake with phase velocity equal to the group velocity of the short pulse, which can be used for particle acceleration. It turns out that, by modulating the amplitude and frequency of the pump, one can generate periodic phase-controlled accelerating structures in the plasma ("plasma linac"). By the judicious choice of the duration of each segment of the pumping beam, acceleration unlimited by the dephasing can be realized. The amount of transverse focusing can be also adjusted. The important advantage of this type of plasma accelerator over the conventional laser wakefield accelerator is that it requires modest laser intensities $I \ll 10^{18} \mathrm{~W} / \mathrm{cm}^{2}$.
\end{abstract}

\section{INTRODUCTION}

Plasma is an attractive medium for particle acceleration [1] because of the high electric field it can sustain. In a plasmabased accelerator particles gain energy from a longitudinal plasma wave. To accelerate particles to relativistic energies, the plasma wave needs to be sufficiently intense, with a phase velocity close to the speed of light. The longitudinal (accelerating) field of the plasma wave is $E_{0}=\hat{n} E_{\mathrm{wb}}$, where $\hat{n}=\delta n / n_{0}$ is the fractional density perturbation of the plasma by the wave, and $E_{\mathrm{WB}}=m c \omega_{p} / e$ is the cold wavebreaking field. Here $\omega_{p}=\sqrt{4 \pi e^{2} n_{0} / m}$ is the plasma frequency, $n_{0}$ is the plasma density, $-e$ and $m$ are the electron charge and mass. In a laser wakefield accelerator (LWFA) such a plasma wave is excited by an ultrashort pulse of about $1 / \omega_{p}$ duration. Since $\hat{n} \approx a_{0}^{2} / 2$, where $a_{0}^{2}=0.37 I_{0} / 10^{18} \mathrm{~W} / \mathrm{cm}^{2}$, high laser intensity $I_{0} \sim 10^{18}$ $\mathrm{W} / \mathrm{cm}^{2}$ is required.

In this paper we suggest a new approach to generating accelerating wakes in plasma, which we call a collidingbeam accelerator (CBA). This method requires neither ultra-high intensity lasers, nor high-current electron beams. Rather, by colliding two counter-propagating laser beams of sub-relativistic intensities, a short timing beam (TB) $a_{0}$ and a long pumping beam (PB) $a_{1}$, a plasma wave with phase velocity $v_{\mathrm{ph}}$ equal to the group velocity of the short

\footnotetext{
* Work was supported by the DOE Division of High Energy Physics contract No. DE-AC02-CHO-3073, and the Deutsche Forschungsgemeinschaft.

† Email: gena@pppl.gov
}

pulse $v_{\mathrm{g}} \approx c$ is generated. This wave induces a fractional density perturbation of the plasma, $\hat{n} \approx \omega_{p} / \omega_{0}$, where $\omega_{0}$ and $\omega_{1} \approx \omega_{0}$ are the laser frequencies. In order to induce a density perturbation of this magnitude, laser intensities should satisfy $a_{0} a_{1} \geq \omega_{p}^{2} / \omega_{0}^{2}$. This condition implies nonrelativistic laser intensities $I_{0,1} \ll 10^{18} \mathrm{~W} / \mathrm{cm}^{2}$, because $\omega_{p}^{2} / \omega_{0}^{2} \ll 1$ for tenuous plasmas.

When two laser pulses collide in plasma, they may exchange photons [2]. The direction of the energy flow between the pulses is governed by the Manley-Rowe relation: the higher-frequency photons back-scatter into the lowerfrequency photons. The recoil momentum is deposited into the plasma electrons. For example, when the pump frequency is higher, $\Delta \omega=\omega_{0}-\omega_{1}<0$, plasma electrons, on average, acquire a negative momentum and produce an electron current. It is essential that this current can generate a plasma wave substantially larger in amplitude (the enhanced wake) than the conventional plasma wake produced by mere forward scattering. Since the sign of the current is controlled by the frequency detuning $\Delta \omega$, so is the phase of the plasma wave $\phi=\omega_{p}\left(t-z / v_{p h}\right)$. These two points are illustrated by a numerical simulation, performed using a 1D version of the Particle-in-Cell (PIC) code VLPL [3]. As Fig. 1 indicates, the addition of the counter-propagating laser beam (pump) to the ultra-short pulse increases the magnitude of the wake by order of magnitude. We will, therefore, refer to the wake generated in the presence of the pump as the "enhanced" wake, as opposed to the "regular" wake, produced in the absence of the pump. Moreover, one observes from Fig. 1 that when the frequency of the pump is changed from $\omega_{1}=1.1 \omega_{0}$ to $\omega_{1}=0.9 \omega_{0}$, the phase of the wake is changed by $\Delta \phi=-\pi$.

The remainder of the paper is organized as follows. In Section 2 we consider the basic physics of the enhanced wake excitation: interference of two counter-propagating laser beams generates a spatially periodic (with period $\lambda_{0} / 2$ ) ponderomotive potential, which can impart an overall momentum to the plasma. Two regimes are considered: when the electron motion in this ponderomotive potential is linear, and when strong electron. In Section 3 we demonstrate how a sequence of acceleration/drift sections can be produced in plasma, mimicking sections of a conventional rf accelerator in the plasma medium.

\section{GENERATION OF ENHANCED WAKES}

We consider the interaction between the electron plasma and two planar circularly polarized laser pulses $\vec{a}_{0}$ and $\vec{a}_{1}$, where $\vec{a}_{0,1}=a_{0,1}\left(\vec{e}_{x} \pm i \vec{e}_{y}\right) / 2, \theta_{0}=\left(k_{0} z-\omega_{0} t\right)$, and 


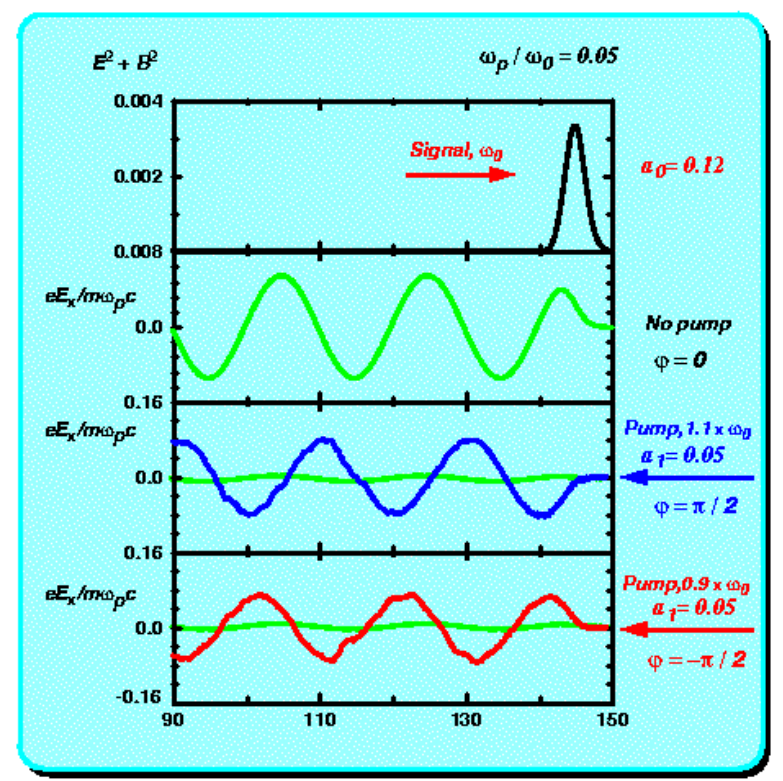

Figure 1: Top to bottom: (i) single short laser pulse with $a_{0}=0.12$ and frequency $\omega_{0}$ propagates from left to right; (ii) short pulse generates a weak plasma wake $E_{x}$; (iii) in the presence of counter-propagating pump with $a_{1}=0.05$ and frequency $\omega_{1}=1.1 \omega_{0}$ the wake is enhanced, and its phase is shifted by $\pi / 2$ with respect to the "regular" wake of (ii), which is also shown for comparison; (iv) Same as (iii), only a down-shifted pump with $\omega_{1}=0.9 \omega_{0}$ is used, and the phase shift is $-\pi / 2$.

$\theta_{1}=\left(k_{1} z+\omega_{1} t\right)$. We assume laser pulses close in frequency $|\Delta \omega| \ll \omega_{0}$, and tenuous plasma $\omega_{p} \ll \omega_{0,1}$, so that $k_{0} \approx k_{1} \approx \omega_{0} / c$.

Plasma electrons experience the longitudinal ponderomotive force of the laser beatwave $F=-m c^{2} \partial_{z} \vec{a}_{0} \cdot \vec{a}_{1} \approx 2 k_{0} a_{0}(\zeta, z) a_{1} \cos \left(2 k_{0} z-\Delta \omega t\right)$, where $\zeta=t-z / v_{g}$. The motion of an arbitrary plasma electron (labeled by index $j$ ) is determined by its ponderomotive phase $\psi_{j}=2 k_{0} z_{j}-\Delta \omega t_{j}$, where $z_{j}$ and $t_{j}$ are the electron position and time, respectively. The equation of motion for the $j$ 'th electron can be expressed as

$$
\frac{\partial^{2} \psi_{j}}{\partial \zeta^{2}}+\omega_{B}^{2} \sin \psi_{j}=-\omega_{p}^{2} \sum_{l=1}^{\infty} \hat{n}_{l} e^{i l \psi_{j}}-\frac{2 \omega_{0} e E_{z}}{m c}+\text { c. c. },
$$

where $\omega_{B}^{2}(\zeta, z)=4 \omega_{0}^{2} a_{0} a_{1}$ is the bounce frequency of an electron in the ponderomotive potential. It turns out that two plasma waves are excited by the collision of a short pulse with a long pump: a slow wave with the wavelength $\lambda_{0} / 2$, and a fast wave (enhanced wake) with the wavelength $\lambda_{p}$. This is reflected in Eq. (1): $\hat{n}_{l}=\left\langle e^{-i l \psi_{j}} / l\right\rangle_{\lambda_{0} / 2}$ is the $l$-th harmonic of the slow wave, and $E_{z}$, the enhanced wake, is the electric field of the fast wave.

The nonlinear origin of the enhanced wake $E_{z}$ can be understood as follows. As the photons are exchanged be- tween the counter-propagating beams, electrons, on average, acquire the recoil momentum and produce a current. However, the current in 1-D must be balanced by the displacement current. An electric field $E_{z}$ is produced, satisfying Faraday's law $\left.\partial E_{z} / \partial t=-4 \pi<J_{z}\right\rangle$, where $\left\langle J_{z}\right\rangle$ is the current averaged over the period of the slow wave. Two flows contribute to $\left\langle J_{z}\right\rangle$ : the linear plasma flow in the field of the enhanced wake $J_{f}=-e n_{0} v_{f}$, and the nonlinear (space-averaged) flow $-e\langle n v\rangle$. Taking the time derivative of Faraday's law, we obtain $\left(\frac{\partial^{2}}{\partial \zeta^{2}}+\omega_{p}^{2}\right) E_{z}=-4 \pi e \frac{\partial<n v>}{\partial \zeta}$.. Below we consider two regimes: when the slow wave is linear and when electrons are trapped by the ponderomotive potential.

If the slow wave remains linear, its higher harmonics can be neglected, and $\langle n v\rangle=n_{0}\left(n_{1} v_{1}^{*}+n_{1}^{*} v_{1}\right)$, where, as defined earlier, $\hat{n}_{1}=\left\langle e^{-i \psi_{j}}\right\rangle$ is the plasma density perturbation, and $v_{1}$ is the associated velocity perturbation. Using the continuity equation, we find that $\langle n v / c\rangle=$ $\left(\Delta \omega / \omega_{0}\right)\left|\hat{n}_{1}\right|^{2}$. It can be shown that behind the Gaussian TB $a_{0}(\zeta)=a_{0} \exp \left(-\zeta^{2} / 2 \tau_{L}^{2}\right)$ the accelerating electric field oscillates as $E_{z}(\zeta)=\tilde{e}\left(m c \omega_{p} / e\right) \sin \omega_{p} \zeta$, where

$$
\begin{aligned}
\tilde{e} & =\frac{\pi \Delta \omega}{8 \omega_{0}}\left(4 a_{1} a_{0} \frac{\omega_{0}^{2}}{\omega_{p}^{2}}\right)^{2} \omega_{p}^{2} \tau_{L}^{2} e^{-\omega_{p}^{2} \tau_{L}^{2} / 4} \times \\
& \times\left[e^{-\left(\omega_{p}-\Delta \omega\right)^{2} \tau_{L}^{2}}+e^{-\left(\omega_{p}+\Delta \omega\right)^{2} \tau_{L}^{2}}+\frac{2}{3} e^{-\Delta \omega^{2} \tau_{L}^{2}}\right]
\end{aligned}
$$

The most efficient excitation of the accelerating wake requires $\tau_{L} \approx 2.0 \omega_{p}^{-1}$ and $\Delta \omega= \pm 1.1 \omega_{p}$. For these parameters $|\tilde{e}| \approx 0.6 \omega_{p} / \omega_{0}\left(4 a_{0} a_{1} \omega_{0}^{2} / \omega_{p}^{2}\right)^{2}$. The enhanced wake exceeds the regular wake from forward scattering whenever $a_{1}>\left(\omega_{p} / \omega_{0}\right)^{3 / 2} / 4$. For $n_{0}=10^{18} \mathrm{~cm}^{-3}$, this corresponds to the pump intensity $I_{1}>2 \cdot 10^{14} \mathrm{~W} / \mathrm{cm}^{2}$.

Equation (2) is valid if the slow plasma wave is linear. To find the maximum magnitude of the enhanced wake, consider the nonlinear regime of Eq. (1) when $\omega_{B}^{2}>\omega_{p}^{2}$. In this regime, all the terms in the RHS of Eq. (1) become smaller than the ponderomotive term in the LHS. One may neglect the electrostatic forces acting on plasma electrons during the short time of TB interaction. Hence, the particle motion is qualitatively described by the nonlinear pendulum equation $\ddot{\psi}_{j}+\omega_{B}^{2}(\zeta) \sin \psi_{j}=0$.

Plasma electrons, initially stationary in the laboratory frame, enter the time-dependent ponderomotive bucket with the initial "speed" $\dot{\psi}=-\Delta \omega$. If this speed is smaller than the bucket height $\dot{\psi}_{\max }=2 \omega_{B}$, some electrons become trapped and execute a synchrotron oscillation in the bucket. It turns out that, by appropriately choosing the pulse duration and frequency detuning, a substantial average momentum can be imparted to plasma electrons. The largest average momentum gain $P_{z} \approx m c \Delta \omega / \omega_{0}$ is realized for $\Delta \omega \approx \omega_{B}$ and $\tau_{L} \approx 2 / \omega_{B}$. For these parameters, most of the electrons execute half of a bounce in the ponderomotive bucket. Behind the TB electrons are left with an average momentum $P_{z}$, generating an enhanced wake 
with the electric field

$$
\frac{e E_{z}}{m c \omega_{p}}=\frac{\left\langle P_{z}\right\rangle}{m c} \sin \omega_{p} \zeta \approx \operatorname{sign}(\Delta \omega)\left(\frac{\omega_{B}}{\omega_{0}}\right) \sin \omega_{p} \zeta
$$

Since the bounce frequency $\omega_{B} \sim I_{0}^{1 / 4}$ increases slowly with the intensity of the TB, it is realistic to assume that $\omega_{B} \sim \omega_{p}$, so that $\hat{n} \sim \omega_{p} / \omega_{0}$. Note that the phase of the enhanced wake is controlled by the sign of the frequency detuning $\Delta \omega$, as observed in the PIC simulation. This is true when the slow plasma wave is either linear, or nonlinear, as predicted by Eqs. (2) and (3), respectively.

\section{PLASMA LiNAC}

The ability to control $\phi$ is important since it may solve the dephasing problem of wakefield acceleration. Dephasing between the plasma wave and the accelerated relativistic electrons occurs after a distance $L_{d}=\lambda_{p}^{3} / \lambda_{0}^{2}$, after which electrons slip into the decelerating phase of the wake. Generating a series of wake sections with tailored relative phases and magnitudes may result in a new type of plasma linac, in which the injected electrons experience acceleration over distances much exceeding $L_{d}$. In order to demonstrate the control over the phase and amplitude of the wake in a CBA, we present in Fig. 1(b) the results of a numerical simulation, where two wake sections of 1 $\mathrm{mm}$ total length and the relative phase difference of $\pi$ are shown. The full dephasing distance of $L_{d}=1 \mathrm{~cm}$ would involve a considerably more computational effort beyond out present capabilities.

The pump is split into two sections: the leading section of duration $\Delta t_{1}=500 \times 2 \pi / \omega_{0}$, where $\Delta \omega=-1.7 \omega_{p}$, and the trailing section $\Delta t_{3}=250 \times 2 \pi / \omega_{0}$, where $\Delta \omega=$ $1.7 \omega_{p}$. These two pump beam sections are separated by the middle section of duration $\Delta t_{2}=\Delta t_{3}$, where the pump is switched out.

As Figs. 2(a,b) show, the three pump sections map into three spatial acceleration regions, which are different from each other by the behavior of the TB, as well as the magnitude and phase of the plasma wake. In the leading region the pump beam has higher frequency and energy flows into the TB, amplifying it. A strong plasma wake with the peak accelerating gradient of $8 \mathrm{GeV} / \mathrm{m}$ is induced. The middle region is void of the pump. Here the TB interacts with the plasma through the usual LWFA mechanism only, producing a weak, $<1 \mathrm{GeV} / \mathrm{m}$, accelerating wake. In this region the energy of the injected electrons does not significantly change, as seen from Fig. 2(d). When the trailing (lowfrequency) part of the pump collides with the TB, the energy flows from the TB into the PB, Fig. 2(c). Again, a strong plasma wake is induced, Fig. 2(b). This wake, however, is shifted in phase by $\Delta \phi=\pi$ with respect to the leading region. As a result, the particles which gained energy in the leading region are decelerated in the trailing region, Fig. 2(d). By repeating the time sequence for the pump, shown in Fig. 2, with the appropriately chosen durations of the pump sections $\Delta t_{1}=2 L_{d} / c$ and $\Delta t_{2}=\Delta t_{3}=L_{d} / c$,
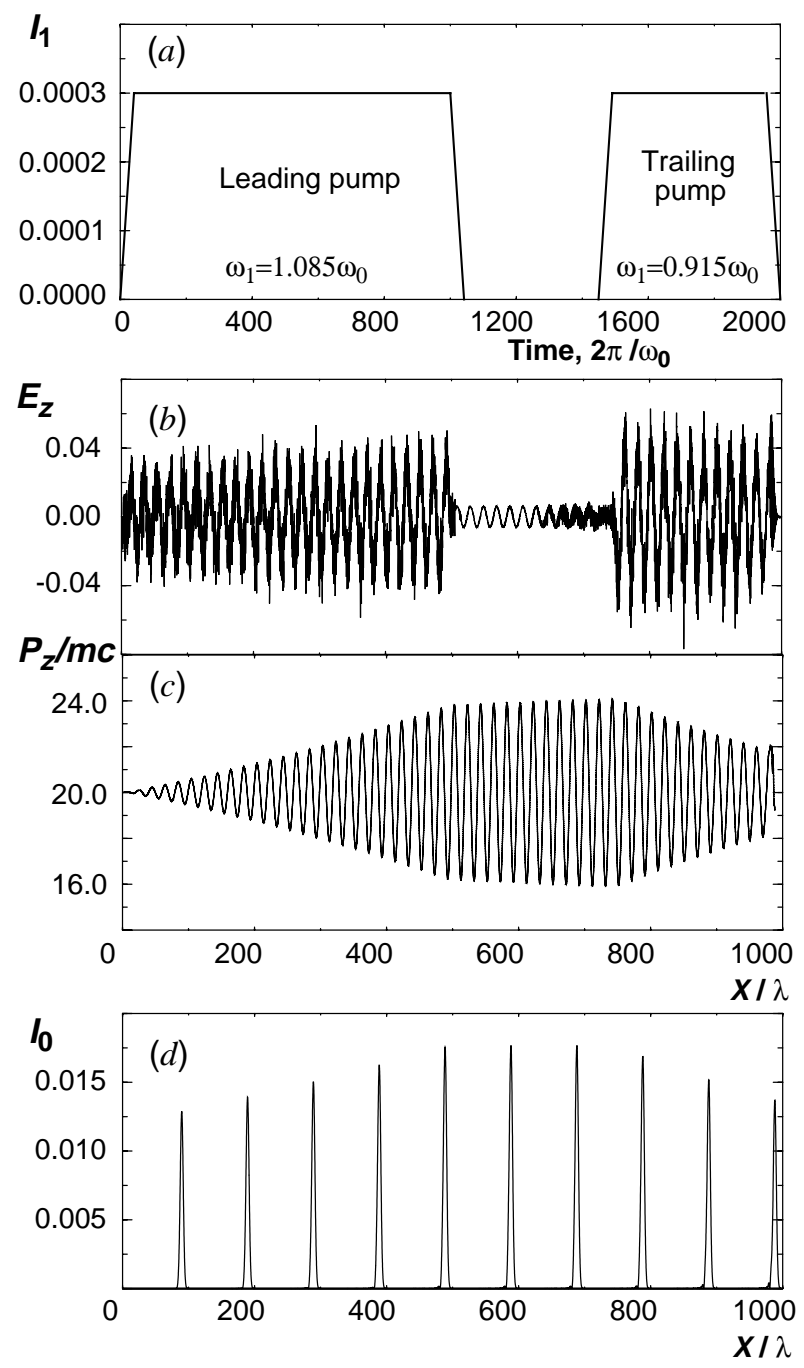

Figure 2: Collision between a short timing beam $\left(a_{0}=\right.$ $\left.0.08, \tau_{L}=\omega_{p}^{-1}\right)$ and an intermittent pump $\left(a_{1}=0.012\right)$ in $n_{0}=2.5 \times 10^{18} \mathrm{~cm}^{-3}$ plasma $\left(\omega_{0} / \omega_{p}=20\right) .10 \mathrm{MeV}$ electrons are continuously injected into the plasma. (a) Timedependence of the pumping beam intensity $I_{1}=2 a_{1}^{2}$; (b) longitudinal electric field $e E_{z} / m c \omega_{p}$; (c) propagation of the TB through the plasma, $I_{0}=2 a_{0}^{2}$; (d) phase space of injected electrons.

one can achieve limitless particle acceleration, not encumbered by dephasing. The convenience of this particular sequence is that the particle is accelerated for $3 / 4$ of the time. Also, since $\Delta t_{1}>\Delta t_{2}$, there is a net energy flow from the pump into the beam which can compensate for the diffractive losses.

\section{REFERENCES}

[1] T. Tajima and J. M. Dawson, Phys. Rev. Lett. 43, 267 (1979).

[2] G. Shvets et. al., Phys. Rev. Lett. 81, 4879 (1998).

[3] A. Pukhov and J. Meyer-ter-Vehn, APS Bulletin 41, 1502 (1996). 